\title{
Interleukin-18 and -10 may be associated with lymph node metastasis in breast cancer
}

\author{
TENG MA ${ }^{1}$ and MENG KONG ${ }^{2}$ \\ ${ }^{1}$ Department of Internal Medicine, The Fifth People's Hospital of Jinan, Jinan, Shandong 250000; \\ ${ }^{2}$ Department of General Surgery, Qilu Children's Hospital of Shandong University, Jinan, Shandong 250022, P.R. China
}

Received August 9, 2020; Accepted January 6, 2021

DOI: 10.3892/ol.2021.12515

\begin{abstract}
Reports on the expression of interleukin (IL)-10 in breast cancer are rare. The present study investigated the correlation between IL-18 and -10 in breast cancer, and assessed their clinical significance. Breast cancer $(n=104)$ and breast fibroadenoma $(n=31)$ tissues that were surgically removed and pathologically confirmed at Jinan Central Hospital Affiliated to Shandong University (Jinan, China) between November 2016 and January 2019 were collected. The expression of IL-18 and -10 was observed via immunohistochemistry. Breast cancer tissues were positive for IL-18 expression, which was primarily located in the cell membrane and cytoplasm. A significant difference in IL-18 expression was observed between breast cancer and fibroadenoma tissues $(75.0$ vs. $19.4 \%$; $\mathrm{P}<0.001)$. IL-10 was expressed in breast cancer tissues and primarily located in the cytoplasm. Breast cancer tissues showed a significantly higher level of IL-10 expression compared with breast fibroadenoma tissues ( 78.8 vs. $22.6 \%$; $\mathrm{P}<0.001)$. The regions of positive IL-18 and -10 expression were consistent. Tissues with positive expression of IL-18 and/or -10 had a significantly higher rate of lymph node metastasis than those with negative expression (IL-18: 67.9 vs. $42.3 \%$; $\mathrm{P}=0.035$; and IL-10: 67.1 vs. $40.9 \%$; $\mathrm{P}=0.047)$. In conclusion, IL-18 is highly expressed in breast cancer and correlates positively with IL-10. Both IL-18 and -10 may correlate positively with lymph node metastasis in breast cancer.
\end{abstract}

\section{Introduction}

Breast cancer is the most common malignancy, which accounted for $\sim 11.6 \%$ of all neoplasms worldwide in 2018 (1), and it accounts for $\sim 15 \%$ of new cancer cases in women, seriously threatening their health and lives (2). Despite great progress in surgery, radiotherapy, chemotherapy and endocrine

Correspondence to: Dr Meng Kong, Department of General Surgery, Qilu Children's Hospital of Shandong University, 23976 Jingshi Road, Jinan, Shandong 250022, P.R. China

E-mail: kongm_jn6346@163.com

Key words: immunotherapy, immunohistochemistry, lymph node metastasis, correlation analysis therapy, the prognosis of advanced and metastatic breast cancer remains poor (3). With the advancement of molecular biology, cell biology and immunology research, biological immunotherapy has become a new therapeutic method for solid tumors, thus altering the treatment mode for a variety of cancer types, such as lung cancer, cancer of the neck and head, renal cancer and malignant melanoma (4). However, for breast cancer, the clinical benefits of immunotherapy remain unsatisfactory, except in a small number of patients with triple-negative breast cancer (5). Exploring the immunological mechanism of breast cancer may provide a new theoretical basis and clinical strategy for the treatment of breast cancer.

Interleukin-10 (IL-10) is a classic immunosuppressive cytokine that plays an important role in regulating the cellular immune response, inhibiting the secretion of proinflammatory factors, and promoting the proliferation and metastasis of tumor cells via immunosuppression (6). IL-10-mediated immunosuppression is realized by synthesizing tumor necrosis factors, IL-1, IL-12 and chemotactic factors, and downregulating the costimulators CD80 and CD86 on the tumor surface (7). IL-10 can also promote the expression and synthesis of IL-6, and induce cellular proliferation by upregulating B-cell lymphoma-2, to change the proliferation and apoptosis of tumor cells; additionally, it inhibits the production of IL-1b, TNF- $\alpha$, IL-6 and MMP-9 in tumors by downregulating vascular endothelial growth factors (8). IL-10 possesses both tumor-promoting and tumor-inhibiting features, and its agonists and antagonists exert therapeutic effects via different mechanisms (9). The immune cells in the tumor microenvironment can secrete a large amount of IL-10, and tumor cells can also produce IL-10. In ovarian cancer, tumor-associated macrophages upregulate the expression of hypoxia-inducible factor $1 \alpha$ by releasing IL-10, which promotes the invasion and metastasis of cancer cells (10). In papillary thyroid carcinoma, the expression of IL-10 is upregulated, which is associated with capsule invasion and lymph node metastasis (11).

IL-18 is a multifunctional cytokine that was first reported in 1995 (12). IL-18 possesses strong immunomodulatory biological activity, exerts anti-infection, antiparasite and antitumor effects by inducing interferon- $\gamma$ (13), promotes perforin- and FasL-mediated cytotoxicity, and directly or indirectly inhibits and destroys malignant tumors via multiple channels (14). Thus, IL-18 inhibits or prevents the growth of breast cancer, bladder cancer and neuroblastoma $(15,16)$. According to a 
previous study (17), IL-18 combined with other cytokines, such as IL-12 and -15 , can regulate the activity of multiple types of immune cells to exert antitumor effects. However, to the best of our knowledge, the correlation between IL-18 and -10 in breast cancer has not been reported.

Based on the aforementioned context, the present study investigated the correlation between IL-10 and -18 in breast cancer, and explored their association with the progression of the disease. The results of this study might provide new insights for the early diagnosis and treatment of breast cancer.

\section{Materials and methods}

Study subjects. The tissue samples used in the present study were obtained from 135 patients with pathologically confirmed breast tumors who underwent surgical resection at the Jinan Central Hospital Affiliated to Shandong University (Jinan, China) between November 2016 and January 2019. Among these patients, 104 had breast cancer. The mean age of the patients with breast cancer was 53.6 \pm 5.2 years (range, 28-69 years). The inclusion criteria were as follows (18): i) No history of malignant tumors; ii) no breast cancer-related treatment, such as neoadjuvant chemotherapy, endocrine therapy, targeted therapy or radiotherapy, before surgery; and iii) complete basic information. In addition, tumor tissues from 31 cases of breast fibroadenoma were used as controls. The ages of these patients ranged from 28-69 years, with a mean age of $54.3 \pm 5.3$ years. The disease courses ranged from 2 months to 8 years, with a mean of $5.9 \pm 1.2$ months. The tumor diameters ranged from $6-20 \mathrm{~mm}$, with 23 cases of unilateral lesions and 8 cases of bilateral lesions. Participants with one or more of the following criteria were excluded from the study (19): Hematological diseases, acute and chronic infections, thyroid diseases, and other benign or malignant tumors. In addition, clinical pathological parameters, such as tumor size, pathological grade, estrogen receptor (ER) status, progesterone receptor (PR) status and HER-2 status, were included in the medical records.

All samples were fixed with $10 \%$ formalin at $25^{\circ} \mathrm{C}$ for $24 \mathrm{~h}$ and embedded in paraffin. Next, $4-\mu \mathrm{m}$ thick sections were cut and conventional hematoxylin and eosin (H\&E) staining and immunohistochemistry were performed. According to the World Health Organization (2003) classification standard (20), among the 104 cases of breast cancer, 23 cases were grade I, 47 cases were grade II and 34 cases were grade III. According to the AJCC (2003) staging criteria (21), 23 cases were stage I, 30 cases were stage IIa, 17 cases were stage IIb, 19 cases were stage IIIa and 15 cases were stage IIIb. Tumor metastasis was based on Tumor-Node-Metastasis staging (22): N0, no metastasis; N1, ispilateral single axillary lymph node metastasis; N2, multiple axillary lymph node metastases; N3, supraclavicular lymph node metastasis; M, distant metastasis.

This research was conducted in accordance with the Declaration of Helsinki and approved by the Ethics Committee of Jinan Central Hospital Affiliated to Shandong University (approval no. 20181103). Written informed consent for research purposes was obtained from each participant.

Main reagents. The main reagents used in the present study included PBS solution (pH 7.4; PBS-0060 phosphate buffer powder), citrate antigen repair buffer (pH 6.0; MVS-0066 citrate buffer), a concentrated DAB color development kit (cat. no. DAB-0031), an EliVision (mouse/rabbit) immunohistochemical kit (cat. no. KIT-9922) (all from Fuzhou Maixin Biotech Co., Ltd.), and rabbit anti-human IL-10 and -18 monoclonal antibodies (BIOSS; cat. no. BS-20373R and BS-4988R, respectively) and poly peroxidase-anti-rabbit/mouse $\mathrm{IgG}$ (Beyotime Institute of Technology; cat. no. P0267).

$H \& E$ staining. Each paraffin block was successively sliced into 5 sections with a thickness of $4 \mu \mathrm{m}$ and then dried at $60^{\circ} \mathrm{C}$ for $60 \mathrm{~min}$. The sections were soaked with xylene I and II, and then subjected to gradient washing with 100, 95, 85 and $75 \%$ alcohol. Afterwards, they were stained with $\mathrm{H} \& \mathrm{E}$ at $25^{\circ} \mathrm{C}$ for $1 \mathrm{~min}$, mounted with neutral resin and then dried. The histodifferentiation of the samples was determined based on the similarity degree between the tumor and the normal tissue according to the pathological section. The more similar the tumor to normal cells indicates a higher degree of differentiation.

Immunohistochemistry. Sections from each paraffin block were dried at $60^{\circ} \mathrm{C}$ for $60 \mathrm{~min}$. After dewaxing with xylene, the samples were rinsed with gradient alcohol solutions, the same procedures as aforementioned. Afterwards, they were repaired with citrate antigen repair buffer $\left(\mathrm{pH} \mathrm{6.0)}\right.$ at $95^{\circ} \mathrm{C}$ for 2 min and then cooled at room temperature. Primary antibody working solution [dilution 1:100 in PBS-BSA (1\% BSA)] at $50 \mu \mathrm{l}$ was applied to each section for incubation at $37^{\circ} \mathrm{C}$ for $90 \mathrm{~min}$. After rinsing in PBS, a secondary antibody (dilution, $1: 1,000)$ was added and the sample was incubated at $37^{\circ} \mathrm{C}$ for $20 \mathrm{~min}$. Finally, the sections were subjected to diaminobenzidine (DAB) coloration, hematoxylin counterstaining $\left(25^{\circ} \mathrm{C}\right.$ for 2 min), dehydration and mounting.

Outcome determination. Each section was read by two pathologists using the double-blind method. Both pathologists were not aware of the objectives of the study. When a count difference of more than $10 \%$ occurred, the pathologists were required to recount. The appearance of brown-yellow coloration or brown-yellow particles in the cell after DAB development was considered to indicate an IL-18-positive result. The outcomes were determined using the semiquantitative integration method (20). Specifically, three fields under a light microscope at x200 magnification were randomly selected, and scores were assigned according to the proportion of positive cells and the degree of staining. For IL-18, 1 point was assigned when $<1 / 3$ of the total cells were stained, 2 points were assigned when $1 / 3$ to $2 / 3$ of the cells were stained and 3 points were assigned when $>2 / 3$ of the cells were stained. According to the degree of coloration, 0 points indicated no coloration, 1 point indicated light yellow coloration, 2 points indicated a brownish yellow coloration and 3 points indicated a brown coloration. For each section, two scores were therefore obtained. The two scores were multiplied to obtain a final score, based on which the positivity of IL-18 in the section was assessed according to the following criteria: 3 points, $(1+)$; $4-5$ points, $(2+)$; and $6-9$ points, $(3+)$. The sections with a total IL-18 score of $\leq 2$ points were considered to be IL-18-negative $(23,24)$. 

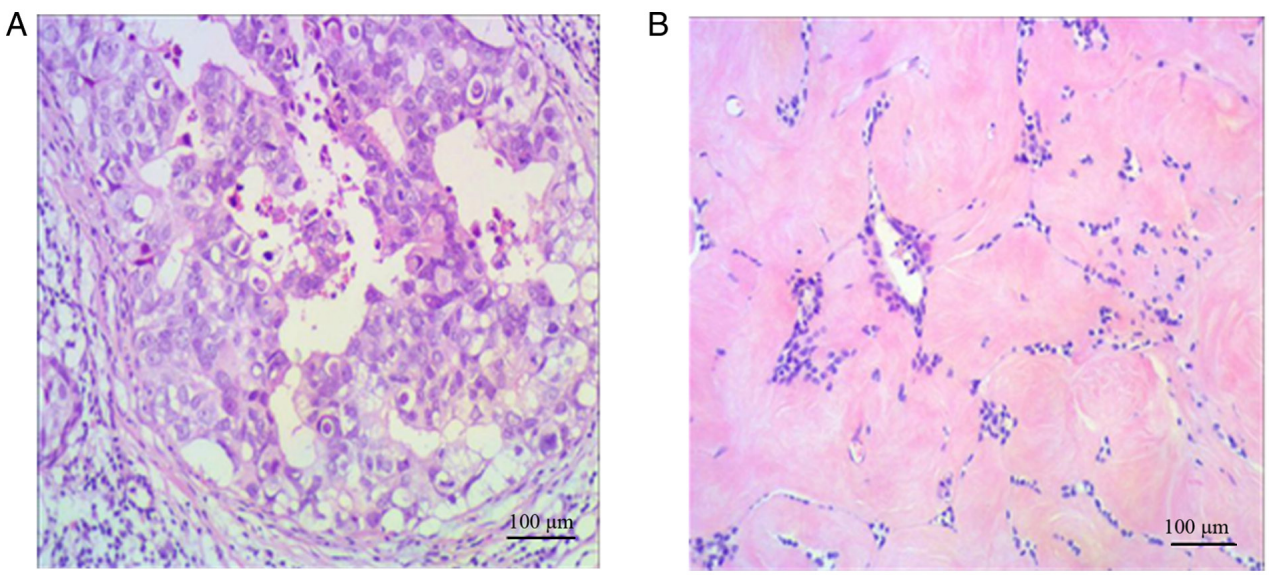

Figure 1. Hematoxylin and eosin staining of breast cancer and breast fibroadenoma tissues (magnification, x200). (A) Breast cancer tissue. (B) Breast fibroadenoma tissue.

The expression of IL-10 was assessed as follows: 0 points, no coloration; 1 point, light yellow coloration; 2 points, coloration between the manifestations of 1 and 3 points; and 3 points, yellow to brownish yellow coloration. The positive cell ratio was scored as follows: 0 points, $0 \%$ staining; 1 point, $1-25 \%$ staining; 2 points, $26-50 \%$ staining; 3 points, $51-75 \%$ staining; and 4 points, $76-100 \%$ staining. The total score of IL-10 was also calculated by multiplying the staining intensity score with the positive cell ratio score, with a score of $\leq 2$ considered to be negative and $\geq 3$ considered to be positive. In addition, colon fibroadenoma tissues from the Department of Gastrointestinal Surgery of Jinan Central Hospital affiliated to Shandong University were used as another negative control for IL-18 and IL-10.

Statistical analysis. Data are presented as percentages, and statistical analyses were performed with SPSS 22.0 (IBM Corp.). The $\chi^{2}$ test was used to compare the groups, and Spearman's correlation analysis was performed to assess the correlation between IL-18 and IL-10. P $<0.05$ was considered to indicate a statistically significant difference.

\section{Results}

$H \& E$ staining. The $H \& E$ staining results of breast cancer and breast fibroadenoma are shown in Fig. 1. The boundaries between the cancer tissue and the surrounding breast tissues were unclear (Fig. 1A). In the breast cancer specimens, the cells were in nest-like or sheet-alike arrangements, with a large amount of intercellular substances. The cells exhibited high-level atypia, and part of the cytoplasm was transparent. A small number of tumor thrombi were observed in the vessels. In the fibroadenoma specimens, the boundaries between the tumor and the surrounding tissues were clear, and the main component of the tumor was proliferative loose fibrous stroma (Fig. 1B). The cells were round or elliptical and grew around the vessel in the form of a vortex or cord. The nuclei did not exhibit heterogeneity. The aggregation and sparsity zones of the cells were alternately distributed. In the stroma, red-stained collagen fibers with different diameters and abundant dendritic thin-walled vessels were found.
Table I. Expression of IL-18 and IL-10 in 104 patients with breast cancer.

\begin{tabular}{|c|c|}
\hline Expression & Patients, \\
\hline IL- $18^{+} / \mathrm{IL}-10^{+}$ & 70 \\
\hline IL-18-/IL-10+ & 12 \\
\hline IL-18+/IL-10' & 8 \\
\hline IL-18-/IL-10- & 14 \\
\hline
\end{tabular}

$\mathrm{IL}$, interleukin.

Expression of IL-18. IL-18 was positively expressed in the majority of the breast cancer tissues (Total staining score $\geq 3$ points), and positive staining was mainly located in the cell membrane and cytoplasm with a diffuse distribution (Fig. 2A). In the majority of the breast fibroadenoma tissues, IL-18 was negatively expressed ( $\mathrm{H} \leq 2$ points; Fig. $2 \mathrm{~B})$. The positive expression rate of IL-18 in breast cancer tissues was significantly higher than that in breast fibroadenoma tissues $[75.0 \%$ (78/104) vs. $19.4 \%$ (6/31); P<0.001; Fig. 2C, D and E]. The negative expression of IL-18 in colon fibroadenoma tissues is shown in Fig. S1A.

Expression of $I L-10$. IL-10 was positively expressed in the majority of the breast cancer tissues ( $\mathrm{H} \geq 3$ points), and positive staining was mainly located in the cytoplasm (Fig. 3A). In the majority of the breast fibroadenoma tissues, the expression of IL-10 was relatively low ( $\mathrm{H} \leq 2$ points; Fig. $3 \mathrm{~B})$. The positive expression rate of IL-10 in breast cancer tissues was significantly higher than that in breast fibroadenoma tissues $[78.8 \%$ (82/104) vs. $22.6 \%$ (7/31); P<0.001; Fig. 3C, D and E]. The negative expression of IL-10 in colon fibroadenoma tissues is shown in Fig. S1B.

Correlation between $I L-18$ and $I L-10$. The samples were divided into the following four categories according to IL-18 and IL-10 expression in the same sample: i) Both IL-18 and IL-10 were expressed positively $(n=70)$; ii) IL-18 expression 

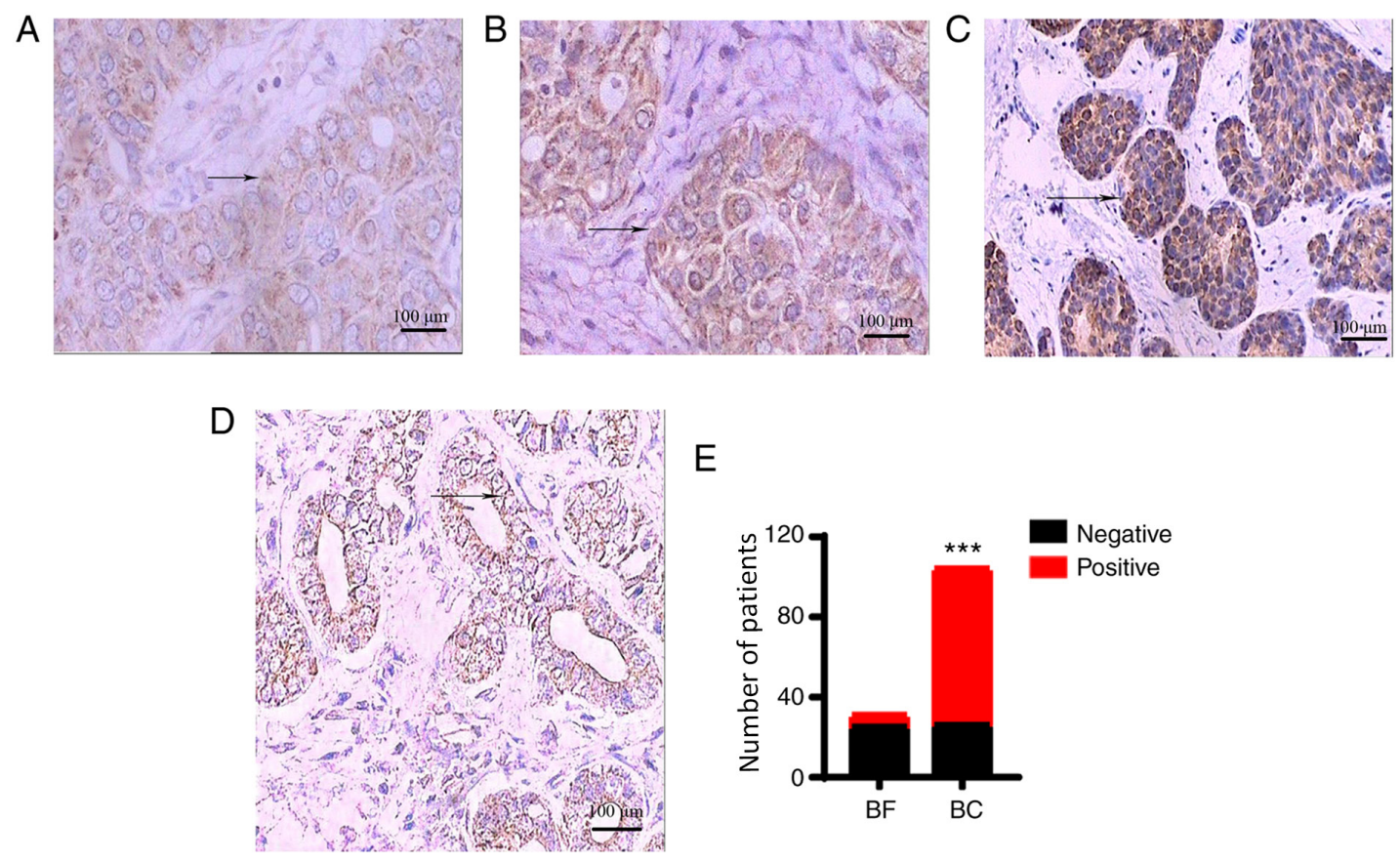

Figure 2. IL-18 expression in tumor tissues (immunohistochemical staining magnification, $\mathrm{x} 200$ ). In breast cancer tissue, the positive expression of IL-18 (arrows) was located in the cell membrane and cytoplasm, with intensities of (A) 1+, (B) 2+ and (C) 3+. (D) Negative or weakly positive expression of IL-18 was observed in the majority of breast fibroadenoma tissues. (E) The positive expression rate of IL-18 in breast cancer tissues was significantly higher than that in breast fibroadenoma tissues. ${ }^{* * *} \mathrm{P}<0.001$ vs. breast fibroadenoma. BF, breast fibroadenoma; BC, breast cancer; IL, interleukin.
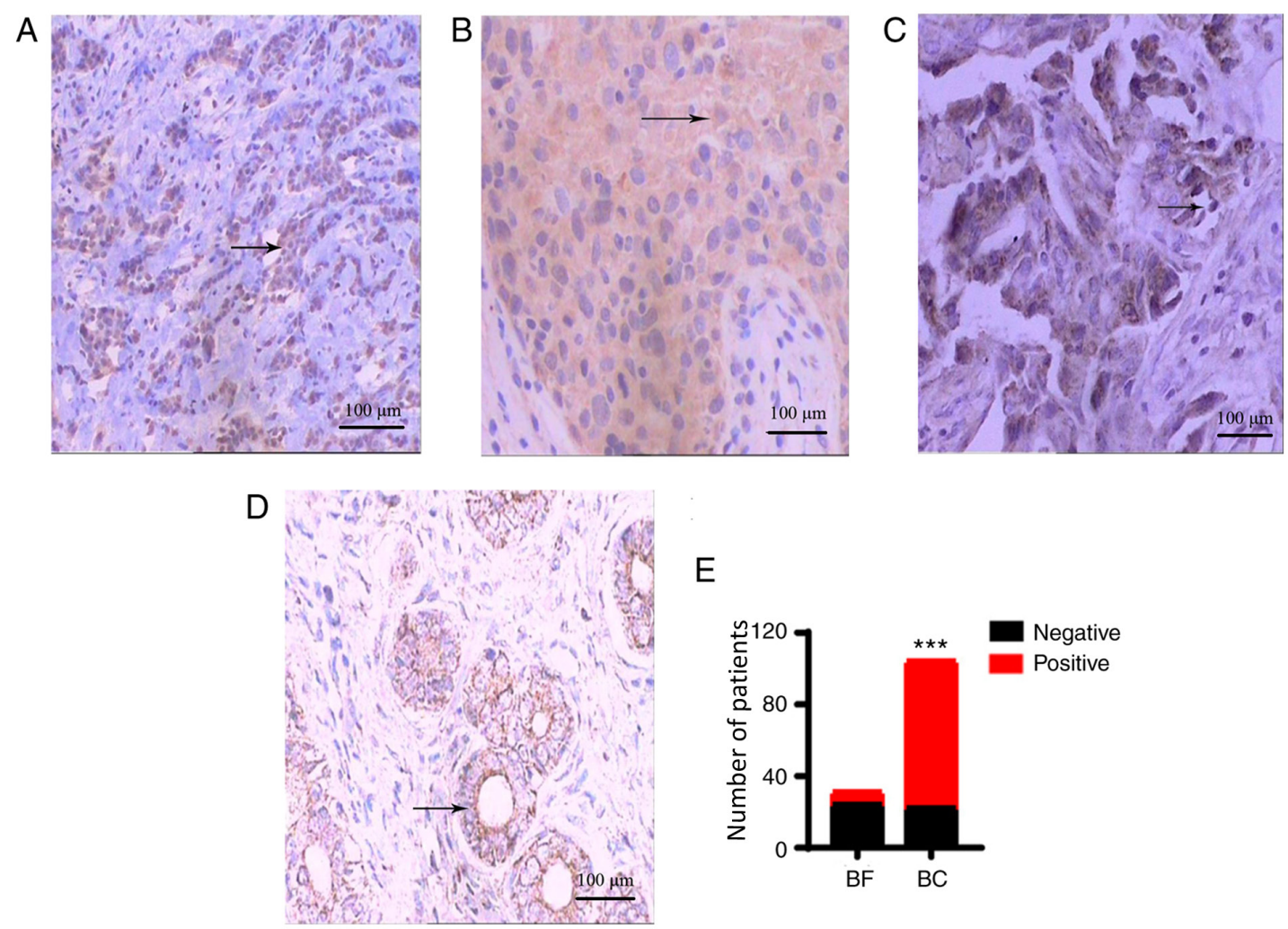

Figure 3. IL-10 expression in tumor tissues (immunohistochemical staining, x100 magnification). In breast cancer tissue, the positive expression of IL-10 (arrows) was mainly located in the cytoplasm with staining intensities of (A) $1+$, (B) $2+$ and (C) 3+. (D) Negative or weakly positive expression of IL-18 was observed in the majority of breast fibroadenoma tissues. (E) The positive expression rate of IL-10 in breast cancer was significantly higher than that in breast fibroadenoma. ${ }^{* * * *} \mathrm{P}<0.001$ vs. breast fibroadenoma. $\mathrm{BF}$, breast fibroadenoma; $\mathrm{BC}$, breast cancer; IL, interleukin.

was negative, while IL-10 was expressed positively $(n=12)$; iii) IL-18 was expressed positively, while IL-10 expression was negative ( $\mathrm{n}=8)$; and iv) IL-18 and -10 expression was negative $(n=14)$ (Table I). Spearman's correlation analysis was 
Table II. Associations of the expression of IL-18 and -10 with clinical pathological indices.

\begin{tabular}{|c|c|c|c|c|c|c|c|}
\hline \multirow[b]{2}{*}{ Index } & \multirow[b]{2}{*}{ Patients, $\mathrm{n}$} & \multicolumn{3}{|c|}{ IL-18 } & \multicolumn{3}{|c|}{ IL-10 } \\
\hline & &,$+ \mathrm{n}$ &,$- \mathrm{n}$ & P-value &,$+ \mathrm{n}$ &,$- \mathrm{n}$ & P-value \\
\hline Age, years & & & & 0.141 & & & 0.093 \\
\hline$\geq 60$ & 32 & 21 & 11 & & 22 & 10 & \\
\hline$<60$ & 72 & 57 & 15 & & 60 & 12 & \\
\hline Max diameter of tumor, $\mathrm{cm}$ & & & & 0.063 & & & 0.820 \\
\hline$>3$ & 40 & 34 & 6 & & 32 & 8 & \\
\hline$\leq 3$ & 64 & 44 & 20 & & 50 & 14 & \\
\hline Histological type & & & & 0.549 & & & 0.283 \\
\hline Infiltrating duct & 86 & 66 & 20 & & 70 & 16 & \\
\hline Lobular & 18 & 12 & 6 & & 12 & 6 & \\
\hline Differentiation degree & & & & 0.579 & & & 0.429 \\
\hline Moderate/high & 82 & 60 & 22 & & 66 & 16 & \\
\hline Low & 22 & 18 & 4 & & 16 & 6 & \\
\hline Staging & & & & 0.809 & & & 0.168 \\
\hline I-II & 70 & 52 & 18 & & 52 & 18 & \\
\hline III-IV & 34 & 26 & 8 & & 30 & 4 & \\
\hline ER & & & & 0.240 & & & 0.328 \\
\hline Positive & 66 & 52 & 14 & & 54 & 12 & \\
\hline Negative & 38 & 26 & 12 & & 28 & 10 & \\
\hline PR & & & & 0.504 & & & 0.148 \\
\hline Positive & 58 & 45 & 13 & & 49 & 9 & \\
\hline Negative & 46 & 33 & 13 & & 33 & 13 & \\
\hline HER-2/neu & & & & 0.282 & & & 0.599 \\
\hline Positive & 80 & 62 & 18 & & 64 & 16 & \\
\hline Negative & 24 & 16 & 8 & & 18 & 6 & \\
\hline Lymph node metastasis & & & & $0.035^{\mathrm{a}}$ & & & $0.047^{\mathrm{a}}$ \\
\hline No & 40 & 25 & 15 & & 27 & 13 & \\
\hline $\mathrm{N} 1-3$ & 64 & 53 & 11 & & 55 & 9 & \\
\hline
\end{tabular}

${ }^{\text {aP }}<0.05$. The expression levels of IL-18 and IL-10 were not associated with age, tumor size, pathological grade (histological type, differentiation level and stage), ER status, PR status or HER-2 status; however, they were associated with lymph node metastasis in breast cancer. ER, estrogen receptor; PR, progesterone receptor; IL, interleukin.

performed according to a previously described method (25). The results showed that the positive expression rate of IL-18 was correlated with that of IL-10 ( $r=0.533$; $\mathrm{P}<0.05$; Fig. 4).

Association of the expression of IL-18 and -10 with lymph node metastasis. The expression of IL-18 and -10 in the lymph node metastasis group was significantly higher than that in the non-lymph node metastasis group (both $\mathrm{P}<0.05$; Fig. 5). However, significant differences were not observed in the age, tumor size, histological type, differentiation degree, clinical grade, ER status, PR status or HER-2 status between these two groups (Table II).

\section{Discussion}

The present study investigated the correlation between IL-18 and -10 in breast cancer, as well as the association of their

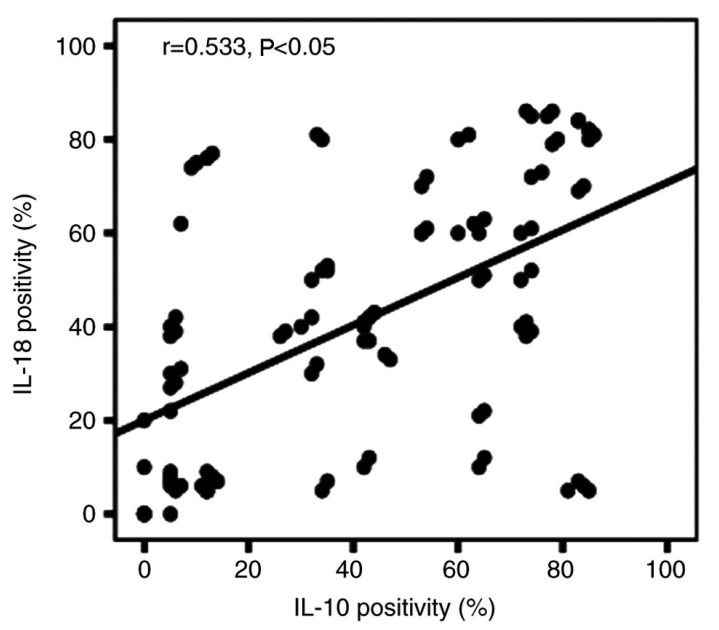

Figure 4. Expression of IL-18 is positively correlated with that of IL-10 in breast cancer according to Spearman's correlation analysis. IL, interleukin. 


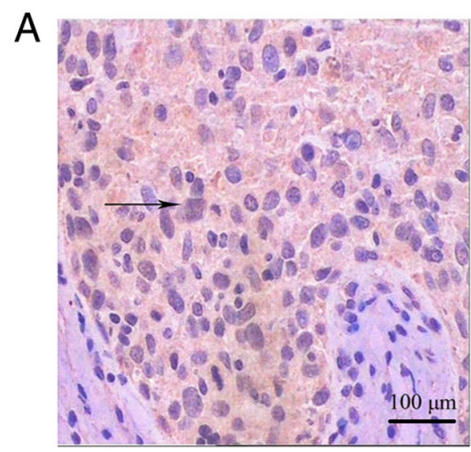

$\mathrm{B}$
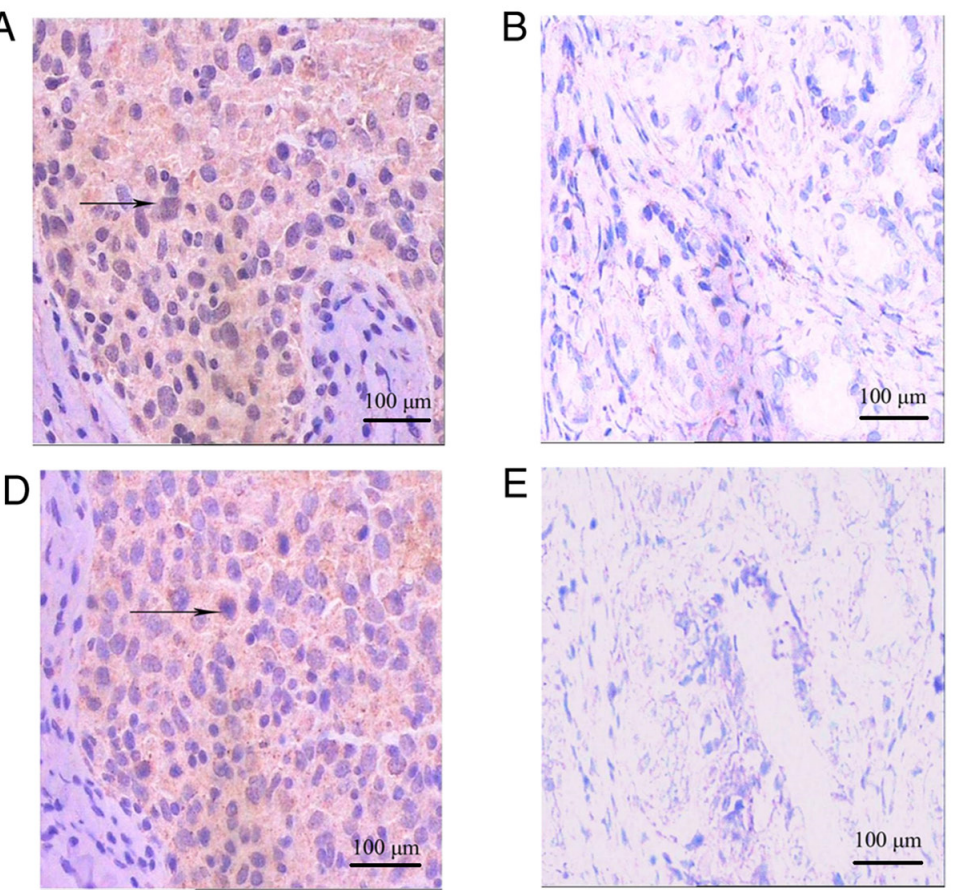

$\mathrm{E}$

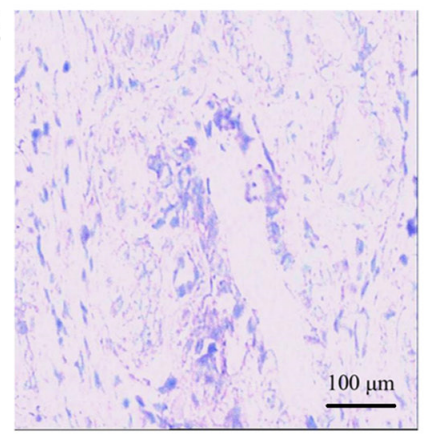

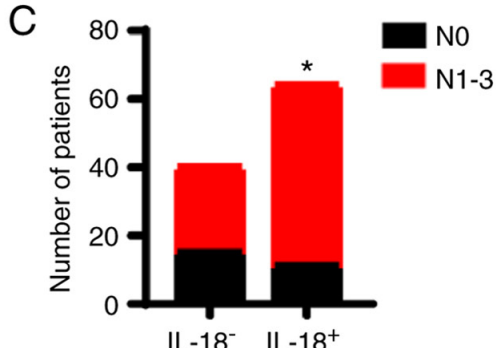

$\mathrm{IL}-18^{-} \quad \mathrm{IL}-18^{+}$
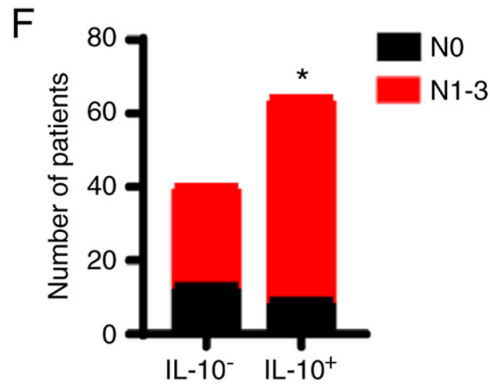

Figure 5. Association of IL-18 and IL-10 positive expression with lymph node metastasis in breast cancer (immunohistochemical staining, $\mathrm{x} 200 \mathrm{magnification).}$ (A) Relatively high expression of IL-18 (arrow) in patients with lymph node metastasis. (B) Relatively low expression of IL-18 in patients with non-lymph node metastasis. (C) Compared with patients with negative IL-18 expression, patients with positive IL-18 expression showed a higher lymph node metastasis rate. (D) Relatively high expression of IL-10 (arrow) in patients with lymph node metastasis. (E) Relatively low expression of IL-10 in patients without lymph node metastasis. (F) Compared with patients with negative IL-10 expression, patients with positive IL-10 expression showed a higher lymph node metastasis rate. ${ }^{*} \mathrm{P}<0.05$ vs. patients with negative expression. IL, interleukin.

expression with the clinical pathological indices of breast cancer.

IL-18 is a multifunctional immunomodulatory cytokine that exists in the form of an inactive precursor; it exerts biological activities only after being sheared by IL-1 $\beta$ invertase (26). In the present study, IL-18 was primarily expressed in breast cancer cells, and it was highly expressed in tumor cells in the majority of the patients with breast cancer. Moreover, its positive expression was associated with lymph node metastasis. By contrast, in benign breast fibroadenoma, IL-18 expression was at low levels or was negative. The level of serum IL-18 in patients with lung cancer, gastric cancer and hepatocarcinoma is significantly higher than that in the healthy population (27). The level of serum IL-18 in patients with breast cancer is also significantly higher than that in the healthy population (28). The present results were consistent with those reported in the literature $(27,28)$. The number of regulatory $\mathrm{T}$ (Treg) cells significantly increases in breast cancer patients with lymph node metastasis, and the accumulation of Treg cells is associated with a short overall survival time in these patients (29). Therefore, the frequency of Treg cells is considered an independent predictor for a high risk of recurrence after breast cancer treatment. IL-18 has been hypothesized to promote the cytotoxicity of Th1 cells, which aggravates the apoptosis of cytotoxic T-lymphocytes (CTLs) and weakens their response mechanisms, thereby promoting tumor growth (17). The present results suggest that high expression of IL-18 in breast cancer cells may serve as a driving factor for the progression of breast cancer and represent an indicator of poor prognosis in patients with this condition.
IL-10 is a protein with a molecular weight of $35-40 \mathrm{kDa}$ that was discovered by Fiorentino in 1989 (30). IL-10 is a multifunctional negative regulator that is primarily secreted by monocytes, helper T lymphocytes (Th2), macrophages and activated B cells; it exerts an immunosuppressive function during malignancy development, which effectively reduces the participation of tumor-immune cytokines, thereby inducing immune escape and promoting tumor growth (31). In the present study, IL-10 was positively expressed in the majority of the breast cancer tissues, but expressed at low levels in the breast fibroadenoma tissues. The positive expression rate in breast cancer tissues was significantly higher than that in breast fibroadenoma tissues. In addition, the study also showed that the expression of IL-10 and -18 in patients with metastatic breast cancer was significantly higher than that in patients without metastasis. A meta-analysis based on 11,170 patients showed that IL-10 was associated with human papillomavirus-related tumors in Asia (32). The level of serum IL-10 in patients with gynecological neoplasms was significantly higher than that in healthy individuals (33). The mechanism underlying this finding may be as follows: Tumor cells, tumor-associated macrophages and regulatory $\mathrm{T}$ cells in the tumor microenvironment are all able to release IL-10, which inhibits the production of IL-2, TNF- $\alpha$ and INF- $\gamma$, thereby suppressing the proliferation and killing ability of T cells $(34,35)$. IL-10 is an immunosuppressive factor that suppresses antitumor immune response by acting upon immunocytes to promote tumor growth and metastasis. IL-10 also upregulates the expression of PDL1 of myeloid cells, which binds with PD1 (an inhibitory receptor of $\mathrm{T}$ cells) to deactivate $\mathrm{T}$ cells, thereby inhibiting 
the antitumor function of T cells $(36,37)$. The present results also suggested that IL-10 may have a tumor-promoting effect in breast cancer tissue. Therefore, it can be used as a diagnostic index of breast cancer; it may also function as a potential target of breast cancer treatment. However, considering that monocytes, Th 2 cells, macrophages and activated B cells are all able to secrete IL-10, further studies are needed to determine the source of IL-10 using the Th2 trace-labeling method (38) to further validate the findings of this study.

According to a previous study (39), IL-18 can function with other cytokines, such as IL-12, to exert tumor-promoting or tumor-suppressing effects. IL-18 may upregulate the expression and activity of NF- $\kappa \mathrm{B}$ to inhibit IL-10, thereby exerting a tumor-suppressing effect (40). Notably, IL-10 can activate the expression of IL-18 through NF- $\kappa \mathrm{B}$ transcription (41). According to Li et al (42), higher expression of IL-18 and -10 in colorectal cancer indicates a higher cancer reoccurrence rate, a poorer prognosis and a shorter survival time, thus indicating the potential as a prognostic indicator for colorectal cancer. Nevertheless, to the best of our knowledge, the regulatory association between IL-18 and -10 has not been reported. The present study found that the expression of IL-10 was positively correlated with that of IL-18 in breast cancer. Although ER, PR and HER-2 are all important clinical parameters associated with breast cancer, the results did not show a noticeable correlation between these parameters and the expression of IL-18 and -10 ( $\mathrm{P}>0.05)$. In addition, the expression of IL-18 and -10 did not show a noticeable correlation with patient age, tumor size or pathological grade $(\mathrm{P}>0.05)$. By contrast, IL-18 and -10 expression was associated with lymph node metastasis in breast cancer. The expression of IL-18 and -10 in the patients with lymph node metastasis (N1-3) was significantly higher than that in the patients without metastasis (N0) $(\mathrm{P}<0.05)$. In addition, the positive expression rates of ER, PR and HER-2 in the metastatic group were noticeably higher than those in the non-metastatic group, which suggested that the positive expression levels of ER, PR and HER-2 may be associated with lymph node metastasis. Thus, the underlying mechanisms may be based on the ability of IL-18 and -10 to regulate each other and their joint participation in the development of breast cancer.

The present study has some limitations. First, all patients included in the study were from the same center and the sample size was small. Therefore, multicenter studies with a larger sample size need to be performed. Second, the patients included in the study received treatment between 2016 and 2019; therefore, the postoperative recurrence and survival of these patients could not be analyzed due to a short follow-up time. In the future, long-term follow-ups for these patients will be conducted to further explore the correlation of IL-18 and -10 with the prognosis of patients with breast cancer. Third, although immunohistochemistry showed that IL-18 and -10 were highly expressed in human breast cancer tissues and lymph node tissues at the protein level and that they were positively correlated, the mechanisms underlying their joint actions were not clear. Last, the present study was preliminary and did not detect the expression of IL-18 and -10 in peritumor tissues or determine the correlations between their expression and clinical outcomes. These issues warrant further research in the future.
In conclusion, IL-10 is highly expressed in breast cancer tissues, and its expression correlates positively with that of IL-18. Both IL-18 and -10 correlate positively with lymph node metastasis in breast cancer, suggesting a possible synergistic effect between them that promotes the development, infiltration and migration of breast cancer. Combined detection of the expression of IL-18 and -10 may provide new indicators for the early diagnosis and prognosis of breast cancer.

\section{Acknowledgements}

Not applicable.

\section{Funding}

The present study was supported by the Health and Family Planning Commission of Jinan (grant no. 2017-2-21).

\section{Availability of data and materials}

The data used and/or analyzed during the current study are available from the corresponding author on reasonable request.

\section{Authors' contributions}

TM designed the study and led the writing of the article. TM and MK conducted the experiments and collected the data. MK conducted the data analysis. TM and MK confirm the authenticity of all the raw data. All authors read and approved the final manuscript.

\section{Ethics approval and consent to participate}

This research was conducted in accordance with the Declaration of Helsinki and approved by the Ethics Committee of Jinan Central Hospital Affiliated to Shandong University, Jinan, China (approval no. 20181103). TM previously worked at Jinan Central Hospital Affiliated to Shandong University and transferred to the Fifth People's Hospital of Jinan (Jinan, China) in July 2020. Written informed consent was obtained from each participant.

\section{Patient consent for publication}

Not applicable.

\section{Competing interests}

The authors declare that they have no competing interests.

\section{References}

1. Ilyas AB, Bahaj RK, Shaikh AA, Khawandanah BS, AI-Foheidi $\mathrm{M}$ and Omer TY: Breast cancer patients' perceptions of their experience with chemotherapy-induced nausea and vomiting and its impact on quality of life in Jeddah Saudi Arabia. Cureus 12: e12038, 2020

2. Bary F, Ferlay J, Soerjomataram I, Siegel RL, Torre LA and Jemal A: Global cancer statistics 2018: GLOBOCAN estimates of incidence and mortality worldwide for 36 cancers in 185 countries. CA Cancer J Clin 68: 394-424, 2018. 
3. Nome ME, Euceda LR, Jabeen S, Debik J, Bathen TF, Giskeødegård GF, Taskén KA, Maelandsmo GM, Halvorsen B, Yndestad A, et al: Serum levels of inflammation-related markers and metabolites predict response to neoadjuvant chemotherapy with and without bevacizumab in breast cancers. Int J Cancer 146 : 223-235, 2020.

4. Zhang B, Zhu S and Song Q: New progress in immunotherapy for breast cancer. Herald Med 38: 1013-1016, 2019.

5. Thakur V and Kutty RV: Recent advances in nanotheranostics for triple negative breast cancer treatment. J Exp Clin Cancer Res 38: 430, 2019.

6. Yuan XH, Li YM, Shen YY, Yang J and Jin Y: Clinical and Th1/Th2 immune response features of hospitalized children with human rhinovirus infection. J Med Virol 92: 26-33, 2020.

7. Ortiz Wilczyñski JM, Olexen CM, Errasti AE, Schattner M, Rothlin CV, Correale J and Silva EA: GAS6 signaling tempers Th17 development in patients with multiple sclerosis and helminth infection. PLoS Pathog 16: e1009176, 2020.

8. Fröschen FS, Schell S, Schildberg FA, Klausing A, Kohlhof H, Gravius S and Randau TM: Analysis of synovial biomarkers with a multiplex protein microarray in patients with PJI undergoing revision arthroplasty of the hip or knee joint. Arch Orthop Trauma Surg 140: 1883-1890, 2020.

9. Sheikhpour E, Noorbakhsh P, Foroughi E, Farahnak S, Nasiri R and Neamatzadeh H: A survey on the role of interleukin-10 in breast cancer: A narrative. Reports Biochem Mol Biol 7: 30-37, 2018.

10. Li Q, Yin RT, Zhou L, Gao Q and Niu YZ: Study on immune adhesins for IL-10 and tumor associated macrophages on the invasion of ovarian cancer. West China Journal of Pharmaceutical Sciences 32: 257-259, 2017.

11. Wang XF, Li J, Lu CH, Wang GQ, Wang ZH, Liu XF, Liu B, Wang G, Zhang Q and Yang Q: IL-10-producing B cells in differentiated thyroid cancer suppress the effector function of $\mathrm{T}$ cells but improve their survival upon activation. Exp Cell Res 376: 192-197, 2019.

12. Shirato K, Imaizumi K, Sakurai T, Ogasawara J, Ohno H and Kizaki T: Regular voluntary exercise potentiates Interleukin-1 $\beta$ and Interleukin-18 secretion by increasing caspase-1 expression in murine macrophages. Mediators Inflamm 2017: 9290416, 2017.

13. Niu XL, Huang Y, Gao YL, Sun YZ, Han Y, Chen HD, Gao XH and Qi RQ: Interleukin-18 exacerbates skin inflammation and affects micro abscesses and scale formation in a mouse model of imiquimod-induced psoriasis. Chin Med J (Engl) 132: 690-698, 2019

14. Zhong DD, Ma HH and Liu JS: Study on correlation between IL-18 level expression and IL-18 gene polymorphism and asthma. Cell Mol Immunol 35: 480-484, 2019.

15. Leifsdottir K, Mehmet H, Eksborg S and Herlenius E: Fas-ligand and interleukin- 6 in the cerebrospinal fluid are early predictors of hypoxic-ischemic encephalopathy and long-term outcomes after birth asphyxia in term infants. J Neuroinflammation 15 : $223,2018$.

16. Zhang W, Dang SQ, Zhang GJ, He HQ and We XP: Genetic polymorphisms of IL-10, IL-18 and IL12B are associated with risk of non-small cell lung cancer in a Chinese han population. Int Immunopharmacol 77: 105938, 2019.

17. Mostafavi E, Esmaeil B and Foroushani SM: Evaluation of cytokines and sialic acids contents in horses naturally infected with theileria equi. Comp Immunol Microb Infect Dis 70: 101453, 2020.

18. Strube F, Infanger M, Wehland M, Delvinioti X, Romswinkel A Dietz C and Kraus A: Alteration of cytoskeleton morphology and gene expression in human breast cancer cells under simulated microgravity. Cell J 22: 106-114, 2020.

19. Brownstone ND, Celie KB, Spigland NA and Otterburn DM: Pediatric breast fibroadenomas: A systematic review and algorithm for treatment. Ann Plas Surg 83: 601-605, 2019.

20. Tavassoli FA and Devilee P: Pathology and Genetics of Tumours of the Breast and Female Genital Organs. World Health Organization Classification of Tumours. Volume IV. IARC Press, Lyon, France, pp 116-119, 2003.

21. Kwan ML, Haque R, Lee VS, Chung WL, Avila CC, Clancy HA, Quinn VP and Kushi LH: Validation of AJCC TNM staging for breast tumors diagnosed before 2004 in cancer registries. Cancer Causes Control 23: 1587-1591, 2012.

22. Jayaprakasam VS, Yeh R, Ku GY, Petkovska I, Fuqua JL III, Gollub $M$ and Paroder V: Role of imaging in esophagea cancer management in 2020: Update for radiologists. AJR Am J Roentgenol 215: 1072-1084, 2020.

23. Liu J, Sun YP, Wang YS, Liu HP and Zhang XL: The significance of expression of B7-H4 in breast cancer. Chin Clin Oncol 12: 830-832, 835, 2017.
24. Dinarvand N, Khanahmad H, Hakimian S, Sheikhi A, Rashidi B, Bakhtiari $\mathrm{H}$ and Pourfarzam M: Expression and clinicopathological significance of lipin-1 in human breast cancer and its association with p53 tumor suppressor gene. J Cell Physiol 235: 5835-5846, 2020.

25. Suzuki S, Dobashi Y, Hatakeyama Y, Tajiri R, Fujimura T, Heldin $\mathrm{CH}$ and Ooi A: Clinicopathological significance of platelet-derived growth factor(PDGF)-B and vascular endothelial growth factor-A expression, PDGF receptor-beta phosphorylation, and microvessel density in gastric cancer. BMC Cancer 10: 659,2010

26. Shiels RG, Hewage W, Pennell EN, Vidimce J, Grant G, Pearson AG, Wagner KH, Morgan M and Bulmer AC: Biliverdin and bilirubin sulfonate inhibit monosodium urate induced sterile inflammation in the rat. Eur J Pharm Sci 155: 105546, 2020.

27. Gao M, Zhang P and Huang L: Is NLRP3 or NLRP6 inflammasome activation associated with inflammation-related lung tumorigenesis induced by benzo(a)pyrene and lipopolysaccharide? Ecotoxicol Environ Safety 185: 109687, 2019.

28. Yang Y, Cheon S, Jung MK, Song SB, Kim D, Kim HJ, Park H, Bang SI and Cho D: Interleukin-18 enhances breast cancer cell migration via down-regulation of claudin-12 and induction of the p38 MAPK pathway. Biochem Biophys Res Commun 459: 379-386, 2015.

29. Hashemi V, Aghebati L, Esmaily M, Masjedi A, Ghalamfarsa G, Namdar A, Yousefi M, Yousefi B and Jadidi-Niaragh F: Regulatory $\mathrm{T}$ cells in breast cancer as a potent anti-cancer therapeutic target. Int Immunopharmacol 78: 106087, 2020.

30. Fiorentino DF, Zlotnik A, Mosmann TR, Howard M and O'Garra A: IL-10 inhibits cytokine production by activated macrophages. J Immunol 147: 3815-3822, 1991

31. Del Giúdice A, Pagura L, Capitani MC, Mainetti LE, Scharovsky OG, Di Masso RJ, Rico MJ and Rozados VR: Nonclassical roles for IFN- $\gamma$ and IL-10 in a murine model of immunoedition. Future Sci OA 6: FSO589, 2020.

32. Qu K, Pang Q, Lin T, Zhang L, Gu ML, Niu WQ, Liu C and Zhang M: Circulating interleukin-10 levels and human papilloma virus and epstein-barr virus-associated cancers: Evidence from a Mendelian randomization meta-analysis based on 11,170 subjects. Onco Targets Ther 7: 1251-1267, 2016.

33. Coosemans AN, Baert T, D'Heygere V, Wouters R, DE Laet L, VAN Hoylandt A, Thirion G, Ceusters J, Laenen A, Vandecaveye $\mathrm{V}$ and Vergote I: Increased immunosuppression is related to increased amounts of ascites and inferior prognosis in ovarian cancer. Anticancer Res 39: 5953-5962, 2019.

34. Mohammad GRKS, Ghahremanloo A, Soltani A, Fathi E and Hashemy SI: Cytokines as potential combination agents with PD-1/PD-L1 blockade for cancer treatment. J Cell Physiol 235: 5449-5460, 2020.

35. Song HS, Liu AZ, Liu GX, Wu F and Li ZT: T follicular regulatory cells suppress Tfh-mediated B cell help and synergistically increase IL-10-producing B cells in breast carcinoma. Immunol Res 67: 416-423, 2019

36. Dennis KL, Blatner NR, Gounari F and Khazaie K: Current status of interleukin-10 and regulatory T-cells in cancer. Curr Opin Oncol 25: 637-645, 2013.

37. Butte MJ, Keir ME, Phamduy TB, Sharpe AH and Freeman GJ: Programmed death-1 ligand 1 interacts specifically with the B7-1 costimulatory molecule to inhibit $\mathrm{T}$ cell responses. Immunity 27 : 111-122, 2007.

38. Lee M, Lee YH, Song J, Kim G, Jo Y, Min H, Kim CH and Park Y: Deep-learning based three-dimensional label-free tracking and analysis of immunological synapses of CAR-T cells. Elife 9: e49023, 2020

39. Hoshino T, Wiltrout RH and Young HA: IL-18 is a potent coinducer of IL-13 in NK and T cells: A new potential role for IL-18 in modulating the immune response. J Immunol 162: 5070-5077, 1999.

40. Al-Tamimi YZ, Bhargava D, Orsi NM, Teraifi A, Cummings M, Ekbote UV, Quinn AC, Homer-Vanniasinkam S and Ross S: Compartmentalisation of the inflammatory response following aneurysmal subarachnoid haemorrhage. Cytokine 123: 154778, 2019.

41. May MJ and Ghhosh S: Signal transduction through NF-kappa B. Immunol Today 19: 80-88, 1998.

42. Li B, Wang F, Ma C, Hao T, Geng L and Jiang H: Predictive value of IL-18 and IL-10 in the prognosis of patients with colorectal cancer. Oncol Lett 18: 713-719, 2019.

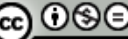

This work is licensed under a Creative Commons Attribution-NonCommercial-NoDerivatives 4.0 International (CC BY-NC-ND 4.0) License. 